\title{
Feasibility study of heavy ion collision physics at NICA JINR
}

\author{
P.N. Batyuk, V.D. Kekelidze, V.I. Kolesnikov, O.V. Rogachevsky, A.S. Sorin, \\ V.V. Voronyuk* on behalf of the BM@N and MPD Collaborations \\ Joint Inst. for Nuclear Research (RU) \\ E-mail: Vadim.Voronyuk@jinr.ru
}

The Nuclotron-based Ion Collider fAcility (NICA) project is under active realization at the Joint Institute for Nuclear Research (JINR, Dubna). The main goal of the project is experimental study of baryon rich QCD matter in heavy ion (up to $\mathrm{Au}$ ) collisions at centre-of-mass energies up to $11 \mathrm{GeV}$ per nucleon. Two modes of the operation is foreseen, collider mode and extracted beams, with the two detectors, MPD and BM@N. In the collider mode the average luminosity is $L=10^{27} \mathrm{~cm}^{-2} \mathrm{~s}^{-1}$ for $\mathrm{Au}(79+)$. Extracted beams of various nuclei species with maximum momenta $13 \mathrm{GeV} / \mathrm{c}$ (for protons) will be available. The NICA project also foresees a study of spin physics with the detector SPD with extracted and colliding beams of polarized deuterons and protons at centre-of-mass energies up to $27 \mathrm{GeV}$ (for protons). The NICA experimental program allows to search for possible signs of the phase transitions and critical phenomena as well as to shed light on the problem of nucleon spin structure. Hereafter, feasibility studies of the observables, which are most sensitive to such phenomena, are presented.

38th International Conference on High Energy Physics

3-10 August 2016

Chicago, USA

${ }^{*}$ Speaker. 


\section{Introduction}

The main goal of physics program at the Nuclotron-based Ion Collider fAcility (NICA) accelerator complex is a search for the mixed phase of quark matter and baryon rich hadronic matter as a consequence of the first order phase transition. Hereafter, we collect the most promising observables which are sensitive to the initial state of the created matter. Full detail of the Monte Carlo analysis of detectors at NICA can be found in publication [1].

\section{Anisotropic flow}

Anisotropic flow is formed at the early stage of collision and is sensitive to the system properties at the very early stage of fireball evolution.

The event plane resolution was estimated from forward hadron calorimeters using a sub-event technique and is shown in Fig.1(left). Good resolution allows to reconstruct direct flow with the high accuracy (Fig.1(middle)). The slope of $v_{1}$ for the identified (by energy loss in the TPC volume)
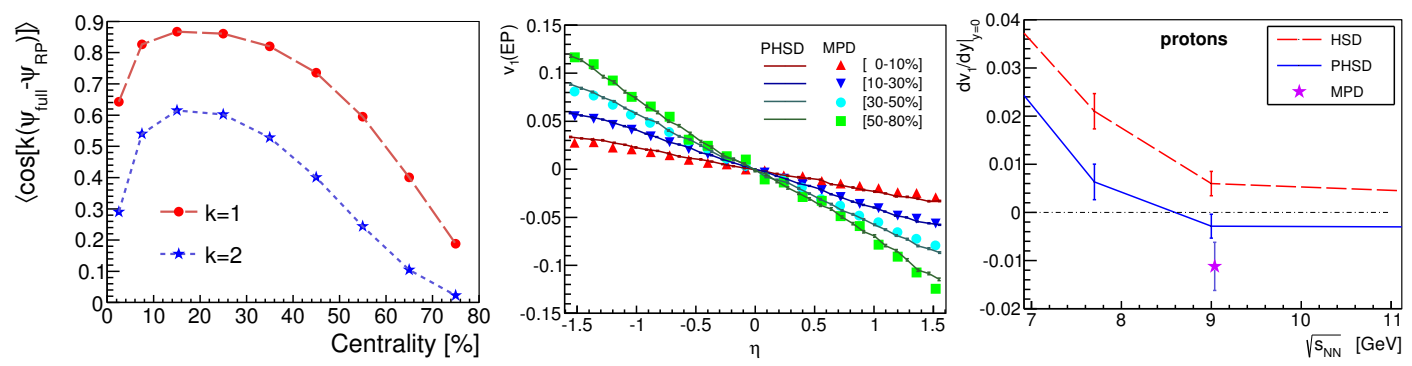

Figure 1: The event plane resolution (left) for the 1st and the 2nd harmonics from the forward hadron calorimeters. The reconstructed direct flow (middle) for charged particles as a function of pseudorapidity and slope (right) of direct flow for protons at mid-rapidity for $\mathrm{Au}+\mathrm{Au}$ collisions at $\sqrt{s_{N N}}=9 \mathrm{GeV}$.

protons from mid-central collisions is shown in Fig.1(right). Also, predictions from the hadronic (HSD) and partonic (PHSD) models are presented there. The observed difference between the reconstructed slope and the model one appears to be due to efficiency of proton identification.

Mixed harmonic technique allows to reconstruct elliptic flow in wide centrality range. Fig.2(left) shows the integrated elliptic flow. The differential distributions of $v_{2}$ for all charged particles for
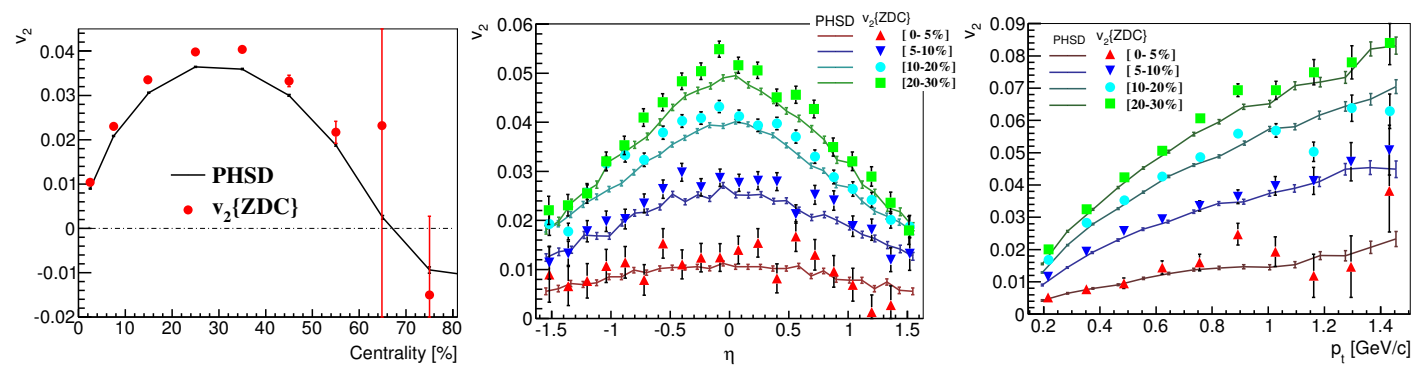

Figure 2: The reconstructed elliptic flow $v_{2}$ for charged particles for Au+Au collisions at $\sqrt{s_{N N}}=9 \mathrm{GeV}$.

several centrality intervals are presented in Fig.2(middle) and Fig.2(right). The reconstructed flow for central and mid-central events reproduces the model distributions slightly overestimating them. In the case of peripheral collisions the obtained error bars are essentially big and increasing of the statistics is required. 


\section{Charged azimuthal correlations}

Charged azimuthal correlations are sensitive to a possible effect of local parity violation (Chiral Magnetic Effect) in the QCD.

Fig.3(left) shows the reconstructed two-particle angular correlation $\gamma_{a b}=\left\langle\cos \left[\phi_{a}+\phi_{b}-2 \Psi_{R P}\right]\right\rangle$ for the same and opposite charged particles with respect to the reaction plane angle $\Psi_{R P}$. It is of in-
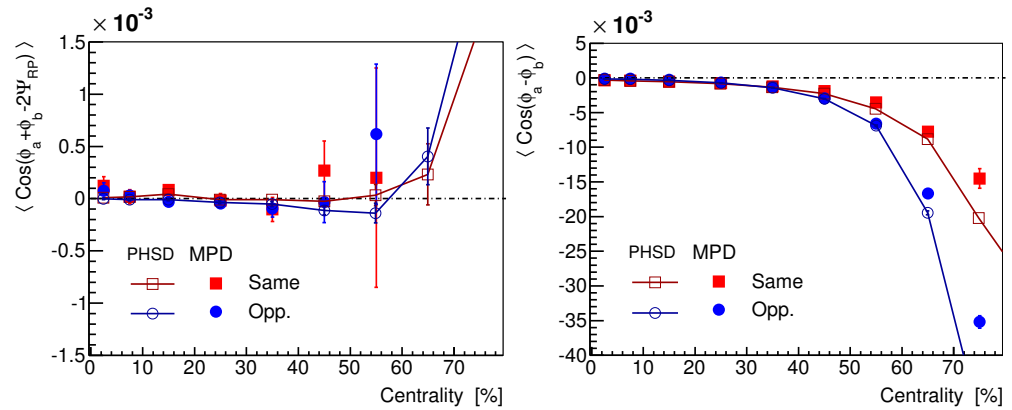

Figure 3: Correlations of the same and opposite charged particles with respect to reaction plane for $\mathrm{Au}+\mathrm{Au}$ collisions at $\sqrt{s_{N N}}=9 \mathrm{GeV}$.

terest to consider the average $\left\langle\cos \left[\phi_{a}-\phi_{b}\right]\right\rangle$ that is supposed to be independent of the reaction plane and can be used in order to estimate systematic errors (Fig.3(right)). The azimuthal correlations and their projections are well reconstructed for central and mid-central collisions.

\section{Baryon stopping power}

Stopping power describes the conversion of the initial collision energy into hot and dense nuclear matter.

The 3-fluid dynamic model (3FD) associates the "wiggle"-behavior of rapidity distribution with a first-order phase transition [2]. To estimate this effect at the NICA energies we use the 3FD model with particlization procedure used for the UrQMD3.4 model [3]. The result of this

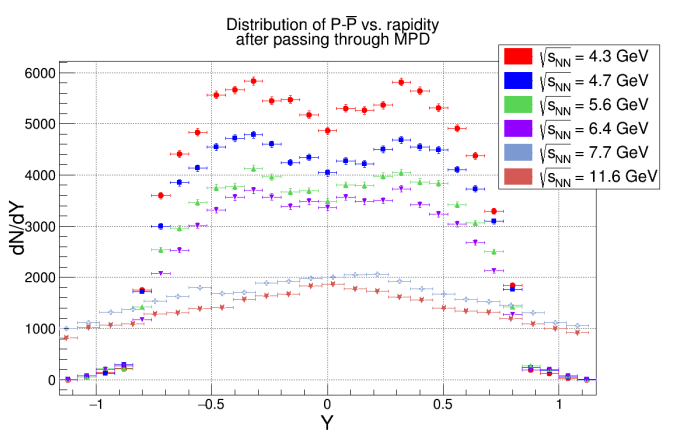

Figure 4: Dependence of the net-proton distribution on rapidity for AuAu-collisions at the NICA energy scan.

simulation shown in Fig.4 clearly indicates such peak-deep-peak behavior at mid-rapidity even with the simulation response for the MPD detector.

\section{Dilepton production}

The correlated lepton production is sensitive to the in-medium properties of hadrons.

The obtained results for the background-subtracted invariant mass distributions of electronpositron pairs and the signal-to-background ratio are shown in Fig 5. The overall signal-to-background ratio was found to be close to $10 \%$ [4]. 

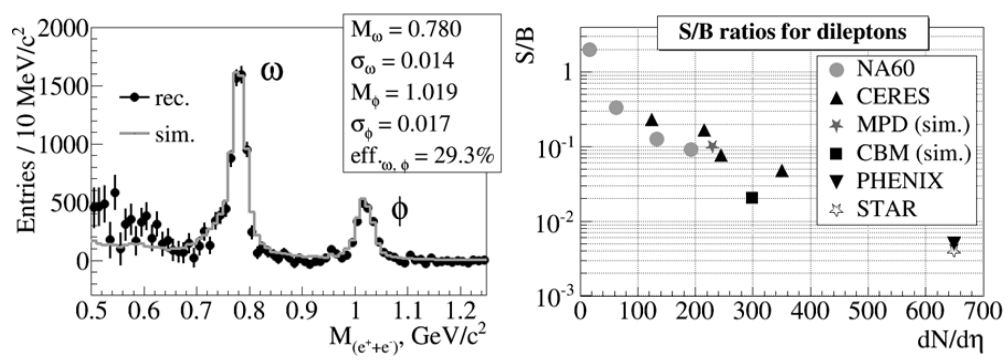

Figure 5: Background-subtracted invariant mass distributions (left) of electron-positron pairs from central $\mathrm{Au}+\mathrm{Au}$ collisions at the MPD. Signal-to-Background (S/B) ratio (right) from heavy-ion experiments as a function of total charged multiplicity.

\section{Hyperon and hypernuclei production}

It is of further interest to consider strangeness production at high baryon densities as well as the mechanism and dynamics of hypernuclei formation.

In Fig. 6 the invariant mass distributions of $\Lambda-\pi^{-}$and $\Lambda-\mathrm{K}^{-}$pairs from central $\mathrm{Au}+\mathrm{Au}$ collisions at the center-of-mass energy $\sqrt{s}=9 \mathrm{GeV}$ are shown. We have estimated the expected yields of particle species under interest for 10 weeks of the data taking at the nominal NICA collider luminosity [5].
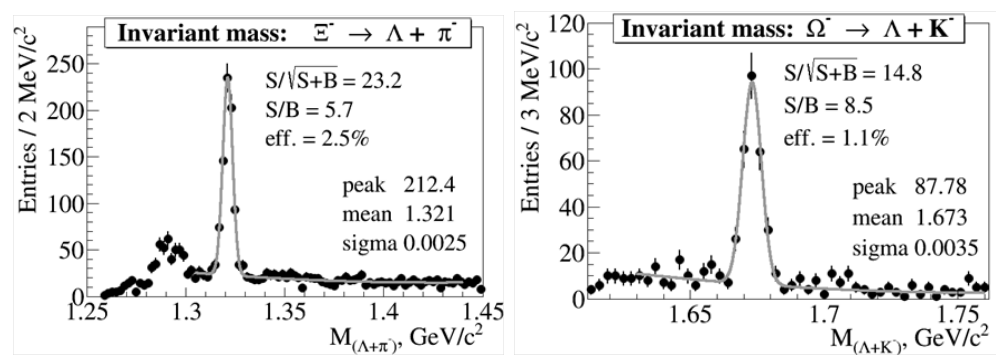

Figure 6: The reconstructed invariant mass of $\Lambda$ and $\pi^{-}$candidates (left) as well as of $\Lambda$ and $\mathrm{K}^{-}$pairs (right) from central $\mathrm{Au}+\mathrm{Au}$ collision at $\sqrt{s_{N N}}=9 \mathrm{GeV}$.

\section{Summary}

The NICA experiments are well suited to provide a variety of experimental data for the critical assessments of the QCD matter phase transitions in the low-temperature and high baryonic density domain of the QCD phase diagram. The sophisticated theoretical models accompanied by results of a realistic Monte Carlo Geant simulation of the proposed experimental setups at NICA support the ability of finding new phenomena in heavy ion collisions.

\section{References}

[1] P.N.Batyuk, V.D.Kekelidze, V.I.Kolesnikov, O.V.Rogachevsky, A.S.Sorin, V.V.Voronyuk Feasibility study of heavy ion physics program at NICA, Phys.Part.Nucl. 47, 4, 540-566 (2016)

[2] Yu.B.Ivanov Alternative Scenarios of Relativistic Heavy-Ion Collisions: II. Particle Production, Phys.Rev. C87, 6, 064905 (2013)

[3] Iu.A.Karpenko, P.Huovinen, H.Petersen, M.Bleicher Estimation of the shear viscosity at finite net-baryon density from $A+A$ collision data at $\sqrt{s_{N N}}=7.7-200 \mathrm{GeV}$, Phys.Rev.C91, 6, 064901 (2015)

[4] V.Vasendina, V.Jejer, V.Kolesnikov et al. Study of the MPD detector capabilities for electron-positron pair measurements at the NICA collider Physics of Particles and Nuclei Letters 10, 7, 769-777 (2013)

[5] M.Ilieva, V.Kolesnikov, Yu.Murin et al. Evaluation of the MPD detector capabilities for the study of the strangeness production at the NICA collider Phys.Part.Nucl.Lett. 12, 1, 100-112 (2015) 